\title{
Dynamic Magneto-mechanical Behavior of an Iron-nickel-based Ferromagnetic Alloy with Constant Elasticity
}

\author{
Leixiang Bian ${ }^{1}$, Yumei Wen ${ }^{1 *}$, Ping $\mathrm{Li}^{1}$, Qiuling Gao ${ }^{1}$, and Xianxue $\mathrm{Liu}^{2}$ \\ ${ }^{1}$ College of Optoelectronic Engineering, Chongqing University, Chongqing City, 400044, P.R. China \\ The Key Laboratory for Optoelectronic Technology \& Systems, Ministry of Education, China \\ ${ }^{2}$ Institute of Electronic Engineering, China Academy of Engineering Physics, Mianyang Sichuan 621900, China
}

(Received 9 October 2008, Received in final form 27 February 2009, Accepted 4 March 2009)

\begin{abstract}
The dynamic magneto-mechanical behaviors in a type of iron-nickel-based ferromagnetic alloy with constant elasticity were investigated as a function of both the DC bias magnetic field $\left(H_{d c}\right)$ and the frequency. The rectangular plate-like samples were excited to vibrate at a half-wavelength, longitudinal resonance by an $\mathrm{AC}$ magnetic field superimposed with various $H_{d c}$. The experimental results found that the strain coefficient at resonance reached $819.34 \mathrm{~nm} / \mathrm{A}$ and the effective mechanical quality factor $\left(Q_{m}\right)$ was greater than 2000 . The ratio of the maximum variation of the Young's modulus over $H_{d c}$ to the value of the Young's modulus at a zero bias field was only $\sim 0.83 \%$ because of the so-called constant elasticity. The resonant strain coefficients and $Q_{m}$ are strongly dependent on $\boldsymbol{H}_{d c}$, which indicates a promising potential for use in DC and quasistatic magnetic field sensing.
\end{abstract}

Keywords : magnetostrictive material, magneto-mechanical behavior, strain coefficient, quality factor

\section{Introduction}

When a ferromagnetic material is placed in magnetic field, it becomes magnetized because the magnetic moments in the material align in such a way that the net magnetic moment points in a particular direction; this realignment of the individual magnetic domains in the material also causes a shift in the material's dimensions, an effect known as magnetostriction [1]. Typical magnetostrictive materials are iron, nickel, cobalt, and their compounds or alloys, such as Fe-Ni, Fe-Co and Fe-Al [2,3], but the effective mechanical quality factors (Q-factor) of these materials are not large [4-8]. The rare-earth-iron alloy Terfenol-D $\left(\mathrm{Tb}_{\mathrm{x}} \mathrm{Dy}_{1-\mathrm{x}} \mathrm{Fe}_{\mathrm{y}}\right)$ features giant magnetostriction and a good magneto-mechanical coupling factor, and so it has generated a great deal of interest over the past few decades. However, the mechanical Q-factor of Terfenol-D is also very small (less than 40) $[9,10]$, and it is difficult to machine Terfenol-D material into small size due to its brittleness.

In this paper, we discuss the dynamic magneto-mechanical behaviors in FeNi-FACE, an iron-nickel-based

*Corresponding author: Tel: +86-23-65112797

Fax: +86-23-65112797, e-mail: ymwen@cqu.edu.cn ferromagnetic alloy with constant elasticity which exhibits a high mechanical Q-factor and low thermal coefficients of Young's modulus and expansion. FeNi-FACE is generally used as an elastic element, a vibrator in mechanical filter, or a resonator. Recently it was discovered that FeNi-FACE exhibits a very high magnetically-induced strain coefficient at resonance. To investigate the dynamic magneto-mechanical behaviors of this material, we applied an AC magnetic field superimposed with various DC bias magnetic fields to rectangular plate-like samples of the material to cause them to vibrate in the half-wavelength $(\lambda / 2)$ longitudinal resonance mode, and we observed the vibration characteristics with a laser doppler vibrometer (LDV). Our experimental results show that the giant strain coefficient in FeNi-FACE results from the high Qfactor. In addition, the resonant strain coefficient and the mechanical Q-factor are strongly dependent on the DC magnetic field. With these results in hand, we discuss the potential application of the FeNi-FACE for DC and quasistatic magnetic field sensing.

\section{Experiments}

Experimental data were obtained from commercially available FeNi-FACE produced by the Chongqing Instru- 
Table 1. The main components and their content percentages in the alloy.

\begin{tabular}{cccccccccc}
\hline \hline Component & $\mathrm{Ni}$ & $\mathrm{Cr}$ & $\mathrm{Ti}$ & $\mathrm{Al}$ & $\mathrm{C}$ & $\mathrm{Si}$ & $\mathrm{Mn}$ & $\mathrm{Fe}$ \\
\hline Content $(\%)$ & $41.5 \sim 43$ & $5.2 \sim 5.8$ & $2.2 \sim 2.8$ & $0.3 \sim 0.8$ & $\leq 0.05$ & $\leq 0.8$ & $\leq 0.8$ & margin \\
\hline
\end{tabular}

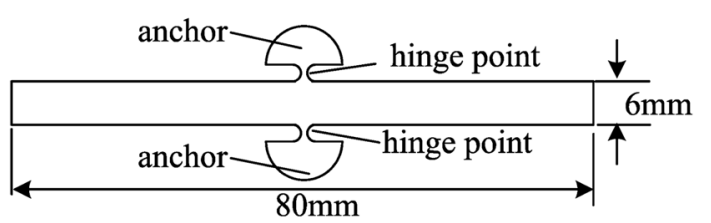

Fig. 1. The architecture of the plate-like samples. The thickness (not shown) is $0.6 \mathrm{~mm}$.

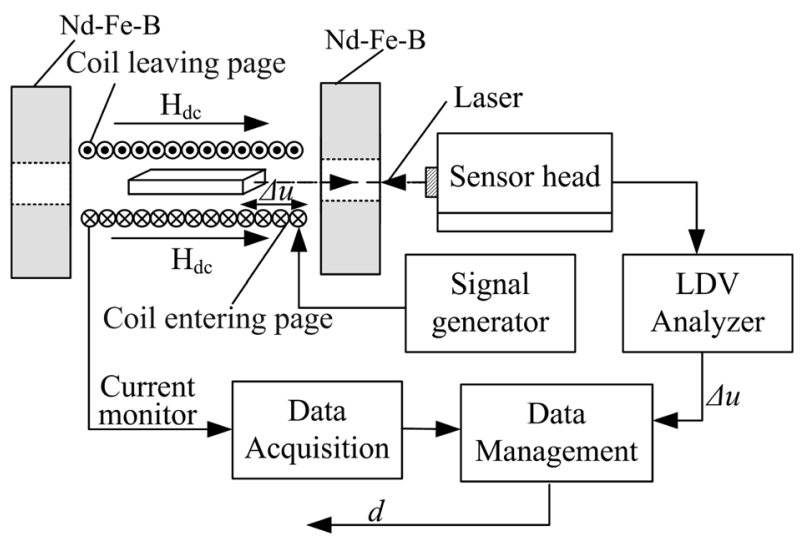

Fig. 2. The experimental setup.

ment Materials Research Institute in China. The main components of the alloy are shown in Table 1. FeNiFACE is a precipitation-hardening alloy. It is provided in the form of ribbons, which for the purpose of this experiment were cut into rectangular plates $6 \mathrm{~mm}$ wide, 80 $\mathrm{mm}$ long, and $0.6 \mathrm{~mm}$ thick, as shown in Fig. 1. At the middle of each rectangular plate were two anchors connected to the plate with micro-hinges, which were integrally machined so as to minimize energy loss to the support mechanism.

The experimental setup for investigating the dynamic magneto-mechanical behavior is shown in Fig. 2. The samples were placed at the center of a long, straight solenoid which generated a small AC magnetic field $\left(H_{a c}\right)$. A bias DC magnetic field $\left(H_{d c}\right)$ was generated with a pair of annular permanent magnets (Nd-Fe-B), and it was measured with a Gauss meter. $H_{d c}$ and $H_{a c}$ were applied along the longitudinal direction of the samples. During the experiments, the samples were driven to vibrate around the $\lambda / 2$ longitudinal resonance by $H_{a c}$ superimposed with $H_{d c}$. The vibrational displacement at the end faces of the samples was measured with an LDV (Polytec OFV-5000, Germany). The experiments were carried out at room temperature and ambient pressure.

\section{Results and Discussion}

In the $\lambda / 2$ longitudinal resonance mode, the strain coefficient of the samples can be calculated by [11]

$$
d=\frac{\Delta \varepsilon}{\Delta H}
$$

where $\varepsilon=2 \Delta u / l$ is the magnetically induced strain, $H$ is the magnitude of the exciting magnetic field, $\Delta u$ is the magnetically induced longitudinal displacement measured by the LDV, and $l$ is the undeformed length of the sample.

Fig. 3 shows the measured strain coefficient as a function of frequency (strain coefficient spectra) for a typical sample near resonance under a magnetic field $H_{d c}=350$. The strain coefficient is found to be $802.5 \mathrm{~nm} / \mathrm{A}$ at the resonance frequency of $\sim 30.650 \mathrm{kHz}$. The effective mechanical Q-factor $\left(Q_{m}\right)$ of the FeNi-FACE plate is given by

$$
Q_{m}=f_{r} / \Delta f,
$$

where $f_{r}$ is the resonance frequency and $\Delta f$ is the 3-dB bandwidth, observed from the strain coefficient spectrum. From Eq. (2), we find the $Q_{m}$ of the FeNi-FACE sample to be 2270 at $H_{d c}=350$. The strain coefficient at resonance is much greater than that of Terfenol-D [10], which is due to the fact that the $Q_{m}$ of the FeNi-FACE is much greater than that of the Terfenol-D. Compared with the static strain coefficient (piezomagnetic coefficient), the strain coefficient at resonance is magnified by the factor of $Q_{m}$. In other words, the resonant strain coefficient is directly proportional to the product of $Q_{m}$ and the piezomagnetic coefficient [9].

Fig. 4 shows the resonant strain coefficient and the

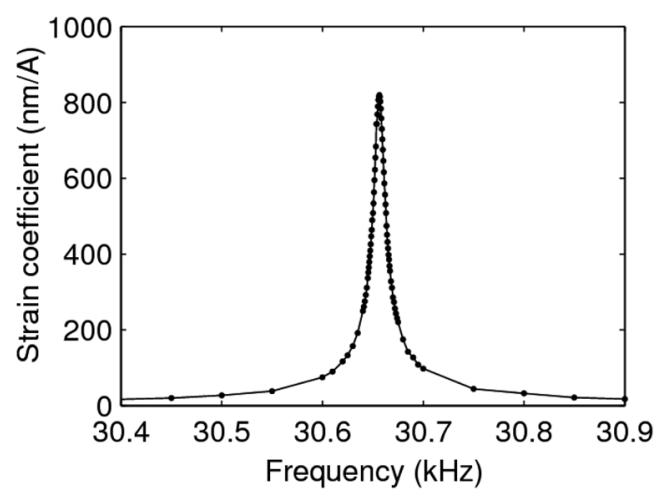

Fig. 3. Strain coefficient spectrum for a typical sample around the resonance. 


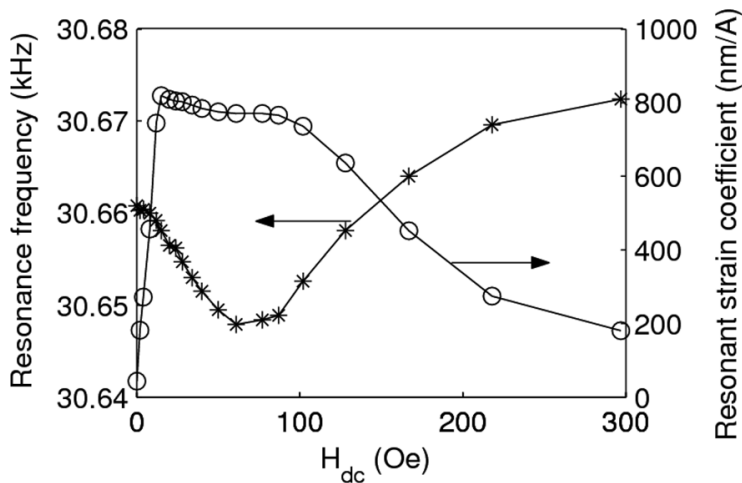

Fig. 4. The resonant strain coefficient and the resonance frequency of the sample as a function of the DC bias magnetic field.

resonance frequency of the sample versus $H_{d c}$. The $H_{d c}$ dependence of the resonant strain coefficient results from the $H_{d c}$ dependence of the piezomagnetic coefficient. In general, with increasing $H_{d c}$ the piezomagnetic coefficient of a magnetostrictive material increases up to a certain maximum and then decreases. From Fig. 4, we can see that the resonant strain coefficient has an almost flat response versus $H_{d c}$ in the range from $15 \mathrm{Oe}$ to $90 \mathrm{Oe}$, which indicates a wide range of optimal $H_{d c}$. This can be understood as a result of the dependence of $Q_{m}$ on $H_{d c}$ (as shown in Fig. 5). As indicated in Fig. 5, the value of $Q_{m}$ varies according to the strain coefficient spectra under various $H_{d c}$. In contrast to the pattern of change of the piezomagnetic coefficient, as $H_{d c}$ increases, $Q_{m}$ first decreases until it reaches a certain minimum and then increases past that point. Taking into consideration the fact that the resonant strain coefficient is directly proportional to the product of $Q_{m}$ and the piezomagnetic coefficient, it is easy to understand the almost flat response characteristic. As Fig. 5 shows, $Q_{m}$ strongly depends on $H_{d c}$. At its point of maximum variation, $Q_{m}$ is only 0.4 of

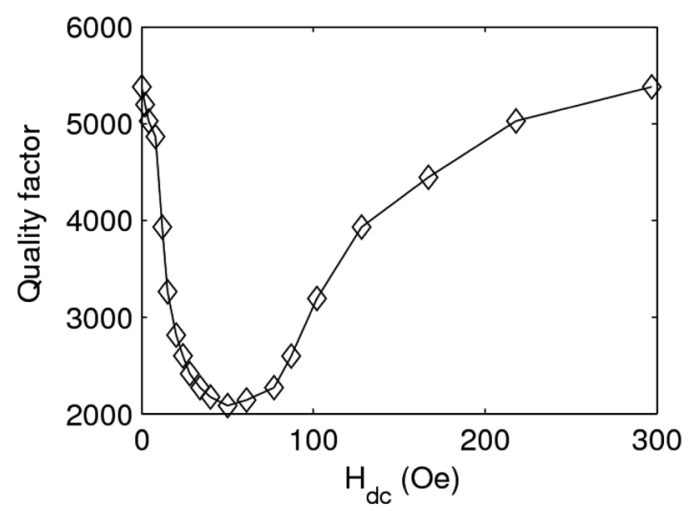

Fig. 5. The effective mechanical Q-factor of the sample as a function of the DC bias magnetic field. the value of $Q_{m}$ at zero bias field, and the differential of $Q_{m}$ with respect to $H_{d c}$ is significantly large.

We can also see from Fig. 4 that the resonance frequency of the sample depends on $H_{d c}$, which can be explained by $\Delta E$ effect [1]. The Young's modulus of FeNi-FACE under various $H_{d c}$ can be derived from the resonance frequency. In the $\lambda / 2$ longitudinal resonance mode, the mechanical resonance frequency is given by

$$
f_{r}=\frac{1}{2 l} \sqrt{\frac{E}{\rho}}
$$

where $\rho=8000 \mathrm{~kg} / \mathrm{m}^{3}$ is the density of FeNi-FACE and $E$ is the Young's modulus at a constant magnetic field along the longitudinal direction. From Eq. (3), we see that the Young's modulus of FeNi-FACE is given by

$$
E=4\left(f_{r} l\right)^{2} \rho
$$

Using the data shown in Fig. 4, we calculate the dependence of Young's modulus on $H_{d c}$, and the results are shown in Fig. 6. As the alloy is a so-called constantelasticity alloy, the $\Delta E$ effect is intrinsically small. As shown in Fig. 6, the maximum variation of Young's modulus as $H_{d c}$ changes is only $0.83 \%$ of the value of the Young's modulus at zero bias field. This characteristic creates high-frequency stability when the FeNi-FACE is used to make a magnetically excited mechanical resonator.

It can be seen from Figs. 5 and 6 that the $Q_{m}$ and Young's modulus change in similar ways with $H_{d c}$, while the resonant strain coefficient changes in the opposite direction. This can be explained by phenomenological theory, as follows. The Q-factor is an inverse measure of damping in a material (or system). The damping arises from the magnetostrictive stress-strain hysteresis loop which is caused by the irreversible motion of magnetic domains and domain walls $[12,13]$. The shapes and areas of the stress-strain hysteresis loops depend on $H_{d c}$ because

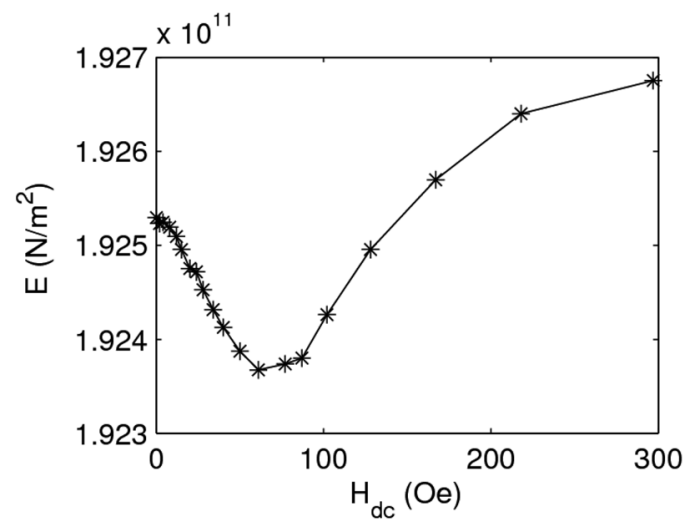

Fig. 6. The Young's modulus of the sample as a function of the DC bias magnetic field. 
of the various domain magnetizing behaviors under various values of $H_{d c}$. This means that the magneto-mechanical damping depends on the DC magnetic field. This hysteresis-related damping has a great influence on $Q_{m}$. The fact that the Young's modulus and the piezomagnetic coefficient depend on $H_{d c}$ can also be traced to the different domain magnetizing behavior under various values of $H_{d c}$ [10]. At low magnetic field values, only the domainwall movement occurs, i.e., the magnetic domains whose moments align with the field grow at the expense of the domains that are not aligned with the field. This results in an initial increase in piezomagnetic coefficient as well as an initial decrease in the Young's modulus as $H_{d c}$ increases. When the $H_{d c}$ is increased further, domain rotation occurs, and the magnetic moments within domains rotate into the easy magnetizing axis closest to the field direction. When $H_{d c}$ is near the so-called "burst region," the rotation of the domains is at a maximum, and the material reaches a maximum piezomagnetic coefficient and a minimum Young's modulus. At higher fields, almost all of the magnetic moments rotate to align with the direction of external field, and rotating the domains becomes difficult, which causes the Young's modulus to increase with increasing $H_{d c}$ and the piezomagnetic coefficient to decrease with increasing $H_{d c}$. The motion of the domain is the major contributor to hysteresis loss. At low and high magnetic field levels, the domain motion is inhibited, but at moderate field intensities the domain moves actively, especially in the burst region. Thus, as $H_{d c}$ increases, $Q_{m}$ decreases at first, up to a certain minimum, and then increases beyond that point.

FeNi-FACE is a potentially feasible material for use in high-sensitivity DC or quasistatic magnetic field sensors. One approach would be to fabricate a magnetoelectric (ME) laminated resonator by combining a piezoelectric material with FeNi-FACE. Since the strain coefficient is intrinsically dependent on $H_{d c}$, such a resonator could detect a small $H_{d c}$ signal by using the operational mode proposed in Ref. [14]. In a result that will be reported elsewhere, we have demonstrated that the ME sensitivity of the ME voltage to $H_{d c}$ achieves $84 \mathrm{mV} / \mathrm{Oe}$ under the resonant drive of a 0.1-Oe AC magnetic field.

Another possible approach would be to fabricate a composite sensor composed of a piezoelectric transformer with FeNi-FACE attached to it on either side (i.e., composite piezoelectric transformer, CPT), as illustrated in Fig. 7. Because of the $H_{d c}$ dependence of the effective mechanical Q-factor $Q_{m}$ in FeNi-FCEA, the loss in the CPT would be dependent on $H_{d c}$. In turn, the output $V_{\text {out }}$ of the CPT would depend on $H_{d c}$. Thus the CPT has the potential to detect small $H_{d c}$ by applying a constant

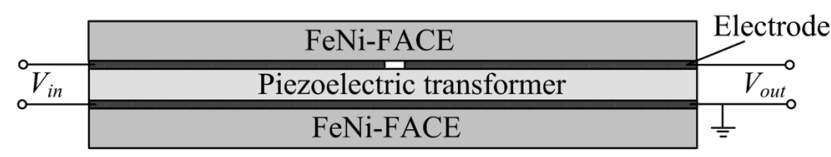

(a)

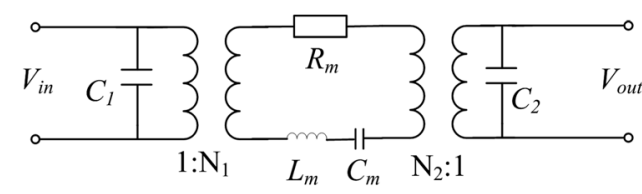

(b)

Fig. 7. A piezoelectric transformer with FeNi-FACE for use in DC magnetic field detection: (a) the configuration of the composite transformer, and (b) the equivalent circuit of the transformer. $R_{m}$ is a lumped representation of all the viscous losses in the CPT. $C_{m}$ and $L_{m}$ are the electric analogs of the effective mechanical spring constant and the mass or inertia of the transformer, respectively. $N_{1}$ and $N_{2}$ are elasto-electric coupling factors, and $C_{1}$ and $C_{2}$ are clamped capacitance in the input and output terminal respectively.

voltage $V_{\text {in }}$ to the input terminal of the CPT. Compared with the method in Ref. [14], this method has the merit of being coil-less.

From the equivalent circuit in Fig. 7(b), we can show that the resonant gain of the transformer is directly proportional to the effective mechanical Q-factor $Q_{\text {eff }}$ of the composite. This gives

$$
V_{\text {out }}=\beta Q_{\text {eff }} V_{\text {in }},
$$

where $\beta$ is a coefficient related to the parameters of the piezoelectric material and of FeNi-FACE. The effective mechanical Q-factor of the CPT $Q_{\text {eff }}$ can be given by [15]

$$
\frac{1}{Q_{e f f}}=\frac{n}{Q_{m}}+\frac{1-n}{Q_{p}},
$$

where $Q_{m}$ is the effective mechanical Q-factor of FeNiFACE, $Q_{p}$ is the effective mechanical Q-factor of the piezoelectric material, and $n$ is the volume ratio of FeNiFACE in the CPT.

Combining (5) and (6) and differentiating $V_{\text {out }}$ with respect to $H_{d c}$, we obtain

$$
\begin{aligned}
& \frac{\partial V_{o u t}}{\partial H_{d c}}=\beta \beta^{\prime} V_{\text {in }} \frac{\partial Q_{m}}{\partial H_{d c}}, \\
& \text { where } \beta^{\prime}=\frac{n}{[n+(1-n) \gamma]^{2}}, \gamma=Q_{m} / Q_{p} .
\end{aligned}
$$

If a piezoelectric quartz crystal with a high Q-factor $\left(>10^{5}\right)$ is used for the piezoelectric transformer element in the CPT, then $\gamma<1$. In this case, it is reasonable to assume that $\beta^{\prime}$ has little influence on the $H_{d c}$ dependence 
of the detection sensitivity under the optimal parameter $n$. The CPT should then show high sensitivity to small changes of $H_{d c}$ because of the strong $H_{d c}$ dependence of $Q_{m}$.

\section{Conclusion}

We investigated the dynamic magneto-mechanical behaviors in FeNi-FACE relative to both the DC bias magnetic field $\left(H_{d c}\right)$ and the frequency of the AC magnetic field $\left(H_{a c}\right)$. The FeNi-FACE displayed a significantly high strain coefficient at resonance and a large effective mechanical Q-factor. Both the strain coefficient at resonance and the effective mechanical Q-factor are strongly dependent on the bias magnetic field. These properties are promising for use in magnetic field sensing.

\section{Acknowledgment}

This work was supported by the National High Technology Research and Development Program of China (863 Program) (No. 2006AA04Z337, 2007AA12Z132) the National Natural Science Foundation of China (No. 50830202, 60774055), and The Joint fund of National Natural Science Foundation of China and the Chinese Academy of Engineering Physics (No. 10776039).

\section{References}

[1] G. Engdahl, Magnetostrictive Materials Handbook, San Diego:Academic Press (2000).

[2] Y. Kikuchi, IEEE Trans. Magn. Mag-4, 107 (1968).

[3] IEEE Standard on Magnetostrictive Materials: Piezomagnetic Nomenclature, IEEE std. 319, (1990).

[4] V. F. Coronel and D. N. Beshers, J. Appl. Phys. 64, 2006 (1988).

[5] D. P. Petarra and D. N. Beshers, J. Appl. Phys. 34, 2739 (1963).

[6] B. F. Rothenstein and Q. F. G. Aczel, J. Appl. Phys. 40, 4675 (1969).

[7] J. Robert Birchak and George W. Smith, J. Appl. Phys. 43, 1238 (1972).

[8] A. Karimi, Ch. Azcoitia, and J. Degauque, J. Magn. Magn. Mater. 215-216, 601 (2000).

[9] F. Claeyssen, N. Lhermet, R. Le Letty, and P. Bouchilloux, J. Alloys Comp. 258, 61 (1997).

[10] S. W. Or, N. Nersessian, and G. P. Carman, Proc. SPIE, 4699, 451 (2002).

[11] S. W. Or, C. S. Yung, and C. Y. Lo, IEEE Trans. Magn. 42, 47(2006).

[12] D. P. Bultea and R. A. Langman, J. Magn. Magn. Mater. 251, 229 (2002).

[13] K. B. Hathaway, A. E. Clark, and J. P. Teter, Metall. Mater. Trans. A 26A, 2797 (1995).

[14] S. X. Dong, J. Y. Zhai, J. F. Li, and D. Viehland, Appl. Phys. Lett. 88, 082907 (2006).

[15] F. Yang, Y. M. Wen, P. Li, M. Zheng, and L. X. Bian, Sens. Actuators A 141, 129 (2008). 